Open Access

\title{
A multi-faceted knowledge translation approach to support persons with stroke and cognitive impairment: evaluation protocol
}

Sara E McEwen ${ }^{1,2^{*}}$, Michelle Donald ${ }^{3}$, Deirdre Dawson ${ }^{2,4}$, Mary Y Egan ${ }^{5}$, Anne Hunt ${ }^{6}$, Sylvia Quant ${ }^{2,3}$, Sharron Runions ${ }^{3}$ and Elizabeth Linkewich ${ }^{2,3}$

\begin{abstract}
Background: Patients with cognitive impairments following a stroke are often denied access to inpatient rehabilitation. The few patients with cognitive impairment admitted to rehabilitation generally receive services based on outdated impairment-reduction models, rather than recommended function-based approaches. Both reduced access to rehabilitation and the knowledge-to-practice gap stem from a reported lack of skills and knowledge regarding cognitive rehabilitation on the part of inpatient rehabilitation team members. To address these issues, a multi-faceted knowledge translation (KT) initiative will be implemented and evaluated. It will be targeted specifically at the inter-professional application of the cognitive orientation to daily occupational performance (CO-OP). CO-OP training combined with KT support is called CO-OP KT. The long-term objective of CO-OP KT is to optimize functional outcomes for individuals with stroke and cognitive impairments. Three research questions are posed:

1. Is the implementation of CO-OP KT associated with a change in the proportion of patients with cognitive impairment following a stroke accepted to inpatient rehabilitation?

2. Is the implementation of CO-OP KT associated with a change in rehabilitation clinicians' practice, knowledge, and self-efficacy related to implementing the CO-OP approach, immediately following and 1 year later?

3. Is CO-OP KT associated with changes in activity, participation, and self-efficacy to perform daily activities in patients with cognitive impairment following stroke at discharge from inpatient rehabilitation and at 1-, 3-, and 6-month follow-ups?
\end{abstract}

Methods/Design: Three interrelated studies will be conducted. Study 1 will be a quasi-experimental, interrupted time series design measuring monthly summaries of stroke unit level data. Study 2, which relates to changes in health care professional practice and self-efficacy, will be a single group pre-post evaluation design incorporating chart audits and a self-report survey. Study 3 will assess patient functional outcomes using a non-randomized design with historical controls. Assessments will occur during admission and discharge from rehabilitation and at 1, 3, and 6 months following discharge from rehabilitation.

Discussion: This project will advance knowledge about the degree to which the implementation of a supported KT initiative can sustainably change health system, knowledge, and patient outcomes.

Keywords: Cognitive orientation to daily occupational performance, Stroke, Cognitive impairment, Rehabilitation, Knowledge translation, Interrupted time series, Protocol

\footnotetext{
*Correspondence: sara.mcewen@utoronto.ca

${ }^{1}$ Sunnybrook Research Institute, Toronto, Canada

${ }^{2}$ University of Toronto, Toronto, Canada

Full list of author information is available at the end of the article
}

(c) 2015 McEwen et al. Open Access This article is distributed under the terms of the Creative Commons Attribution 4.0 International License (http://creativecommons.org/licenses/by/4.0/), which permits unrestricted use, distribution, and reproduction in any medium, provided you give appropriate credit to the original author(s) and the source, provide a link to the Creative Commons license, and indicate if changes were made. The Creative Commons Public Domain Dedication waiver (http://creativecommons.org/publicdomain/zero/1.0/) applies to the data made available in this article, unless otherwise stated. 


\section{Background}

Patients with cognitive impairments following a stroke are often denied access to inpatient rehabilitation [1], despite evidence of its benefits for them [2]. These patients comprise up to $30 \%$ of stroke patients [3]. In the instances when they are admitted to inpatient stroke rehabilitation, they generally receive services based on outdated impairment-reduction models, rather than recommended function-based approaches [4]. These two issues, reduced access to rehabilitation and the knowledge-to-practice gap, both stem from a reported lack of skills and knowledge on the part of stroke rehabilitation teams [4]. To address these issues, we will implement and evaluate a multi-faceted, supported, integrated knowledge translation (KT) initiative, targeted specifically at the inter-professional application of the cognitive orientation to daily occupational performance (CO-OP) approach [5]. The CO-OP approach is a contemporary, effective, cognitive strategy-based treatment approach aligned with Canadian stroke best practice recommendations for cognitive rehabilitation [6]. The long-term objective of this knowledge translation initiative is to optimize functional outcomes for individuals with cognitive impairments following a stroke. Three outputs are expected: (1) increased proportion of patients with cognitive impairments admitted to inpatient stroke rehabilitation; (2) enhanced capacity of inter-professional stroke rehabilitation team members to implement a cognitive-strategy based treatment approach; and (3) improved immediate and long-term functional outcomes for patients with cognitive impairments discharged from inpatient stroke rehabilitation.

This project is a collaboration between a group of knowledge users, lead by Ms. Elizabeth Linkewich, and a group of researchers, lead by Dr. Sara McEwen. Lead knowledge user and decision maker, Ms. Linkewich, is one of three regional directors within the Toronto Stroke Networks (TSNs), an organization charged with overseeing service delivery is in line with best practices and optimization of resources [7]. Ms. Linkewich has convened a knowledge user team of educators, a community re-engagement specialist, and management collaborators. To ensure an integrative KT strategy, these partners have been involved with every aspect of this project's design, beginning with pre-project work to identify the problem and develop plans to address it.

The Toronto Stroke Networks developed a model to aid discharge from acute stroke care in a way that would maximize both patient outcomes and the efficient use of scarce rehabilitation resources, considering severity and patient characteristics [1]. The model indicates that $40 \%$ of patients discharged from acute care should be transferred to specialized inpatient rehabilitation. However, in recent years, the actual proportion has ranged from 26 to $33 \%$, and the discrepancy comes in part from patients with cognitive impairment. Lack of access to inpatient rehabilitation may result in patients being transferred to assisted living facilities without the benefit of rehabilitation that could potentially have enabled them to return home. It may also contribute to the relatively poorer outcomes of patients with cognitive impairments, including higher rates of dependency and disability and lower mood and quality of life [8].

Ontario's Ministry of Health and Long-Term Care and the TSNs have embedded access to inpatient rehabilitation for patients with cognitive impairment into care expectations [9]. However, inter-professional team members' perceived lack of skills to support patients with cognitive impairment remains a major barrier to access to care. Interviews with professionals from Toronto-based rehabilitation teams revealed two important and related knowledge to practice gaps [4]. First, team members reported that they did not have the specialized knowledge and skills to work with stroke patients who were experiencing cognitive impairment. Second, when treating patients with cognitive impairments, team members reported using impairment-reduction models, considered to be outdated, rather than currently recommended function-based models that incorporate problem solving and strategy training. Contemporary function-based models are substantially better than pure impairment reduction models, in that they are associated with sustained improvements in everyday functioning, self-evaluation, and problem solving, among several other benefits [2]. Canadian Stroke Best Practice Recommendations state that interventions should be tailored to meet meaningful, functional patient goals [6]. The CO-OP approach aligns with these recommendations, and has demonstrated efficacy to improve function in people with stroke [10-12], including those with demonstrated cognitive impairment [12-14].

CO-OP, a functional, patient-goal-centered, problemsolving approach, is associated with improved function, activity performance, participation, and self-efficacy in people with stroke $[10,11,13]$, and has demonstrated better efficacy than control conditions $[12,14,15]$. Table 1 provides an overview of relevant $\mathrm{CO}-\mathrm{OP}$ stroke publications. $\mathrm{CO}-$ OP has seven key features that include client-chosen goals, dynamic performance analysis, cognitive strategy use, and guided discovery. In the first session, the patient and a rehabilitation clinician work together, using the Canadian occupational performance measure (COPM) [16], to select personally-valued activities which the patient needs or wants to do. These then form the basis of their rehabilitation goals and become the direct focus of the intervention. In the second session, the patient is taught a global cognitive strategy (Goal-Plan-Do-Check). This strategy is used in an iterative manner in all future sessions as the main problem-solving framework to facilitate skill acquisition/ goal attainment. The patient identifies a goal, is guided by 
the clinician to discover a plan to achieve the goal, does the plan, and finally checks to see if the plan was implemented and if it worked to achieve the goal. Within the plan phase, the clinician uses guided discovery rather than explicit instruction to help the patient analyze the task to be performed and to discover individual strategies that are specific to the particular performance problems of that patient with that chosen activity. A detailed description of CO-OP's theoretical foundations, key features, and administration procedures is available in a publication by Polatajko and Mandich, 2004 [5].

$\mathrm{CO}-\mathrm{OP}$ has an established training infrastructure with available local trainers, and therefore is feasible and efficient to implement. However, given the multi-institutional environment, the need for shifts in attitudes and beliefs (the CO-OP approach requires therapists to give up a significant degree of control to the clients) and the relative ineffectiveness of passive KT strategies [17], CO-OP training by itself is unlikely to be sufficient to cause widespread, sustained practice change. Thus, we will implement a multi-faceted KT initiative that will combine institution-specific support, multi-sectoral collaboration, and managerial participation along with the established $\mathrm{CO}-\mathrm{OP}$ training. These additional health system components are important in moving evidence to practice in complex environments, particularly when shifts in culture, attitudes, and behavior are required [18, 19]. Components of the project will integrate seamlessly with existing TSNs' education and KT infrastructure, and will incorporate online components, including a virtual community of practice. These aspects will facilitate sustainability.

The knowledge to action framework developed by Graham et al. provides the foundation for this project [20]. The knowledge to action framework consists of a central knowledge creation cycle and a concurrent action cycle. Figure 1 depicts the knowledge to action framework with content specific to the CO-OP KT project. In preparatory work, issues were identified and knowledge was synthesized to develop strategies to mitigate those issues. The results led to development of the current project which begins with the phase of adapting the knowledge to the local context and identifying barriers to knowledge uptake, and will continue with selecting, tailoring, and implementing KT intervention, monitoring and sustaining knowledge use, and evaluating outcomes.

Also informing the project are the inter-professional collaboration and inter-professional education constructs. Inter-professional collaboration incorporates effective working relationships among health care providers from different disciplines and their patients and enables optimal health outcomes by building on the foundational elements of "respect, trust, shared decision making, and partnerships" [21]. Inter-professional education emphasizes providers from multiple differences learning with, from, and about each other, and is interdependent with interprofessional collaboration.

Through this project, we expect to see improved knowledge and practice change in inter-professional stroke rehabilitation teams, manifested as greater implementation of cognitive rehabilitation best practices and greater self-efficacy to do so. Following directly from positive practice change, we also expect to see improvements at the health system and health outcome levels. Individual rehabilitation professionals and teams as a whole will come to see themselves as having the specialized knowledge and skills to facilitate functional improvement in patients with cognitive impairments. With this, the number of patients with cognitive impairment who are accepted to inpatient rehabilitation will increase. Further, because previous research indicates that CO-OP is associated with improvements in functional independence, activity performance, self-efficacy, and participation, including in those with demonstrated cognitive impairment [10-15, 22, 23], we expect to see similar positive patient outcomes. Aligning with the three expected project outputs outlined previously in the manuscript, three specific research questions are posed:

1. Is the implementation of CO-OP KT associated with a change in the proportion of patients with cognitive impairment following a stroke accepted to inpatient rehabilitation?

2. Is the implementation of CO-OP KT associated with a change in rehabilitation clinicians' practice, knowledge and self-efficacy related to implementing the CO-OP approach, immediately following and 1 year later?

3. Is CO-OP KT associated with changes in activity, participation, and self-efficacy to perform daily activities in patients with cognitive impairment following stroke at discharge from inpatient rehabilitation and at 1-, 3-, and 6-month follow-ups?

\section{Methods/Design}

To answer the three questions, three interrelated studies will be conducted at five inpatient rehabilitation units in the Greater Toronto Area. See Fig. 2 for a visual depiction of all three studies and expected timeline. The first question, which relates to changes at the health system level, will be addressed using a quasi-experimental, interrupted time series design (Study 1); the second, which relates to changes in health-care professional knowledge, practice, and selfefficacy, will be addressed using a single group pre-post evaluation design (Study 2); and the third, which relates to patient outcomes, will be addressed using a nonrandomized design with historical controls (Study 3). Table 2 provides an overview of outcomes, indicators, and their timing for all three studies. 


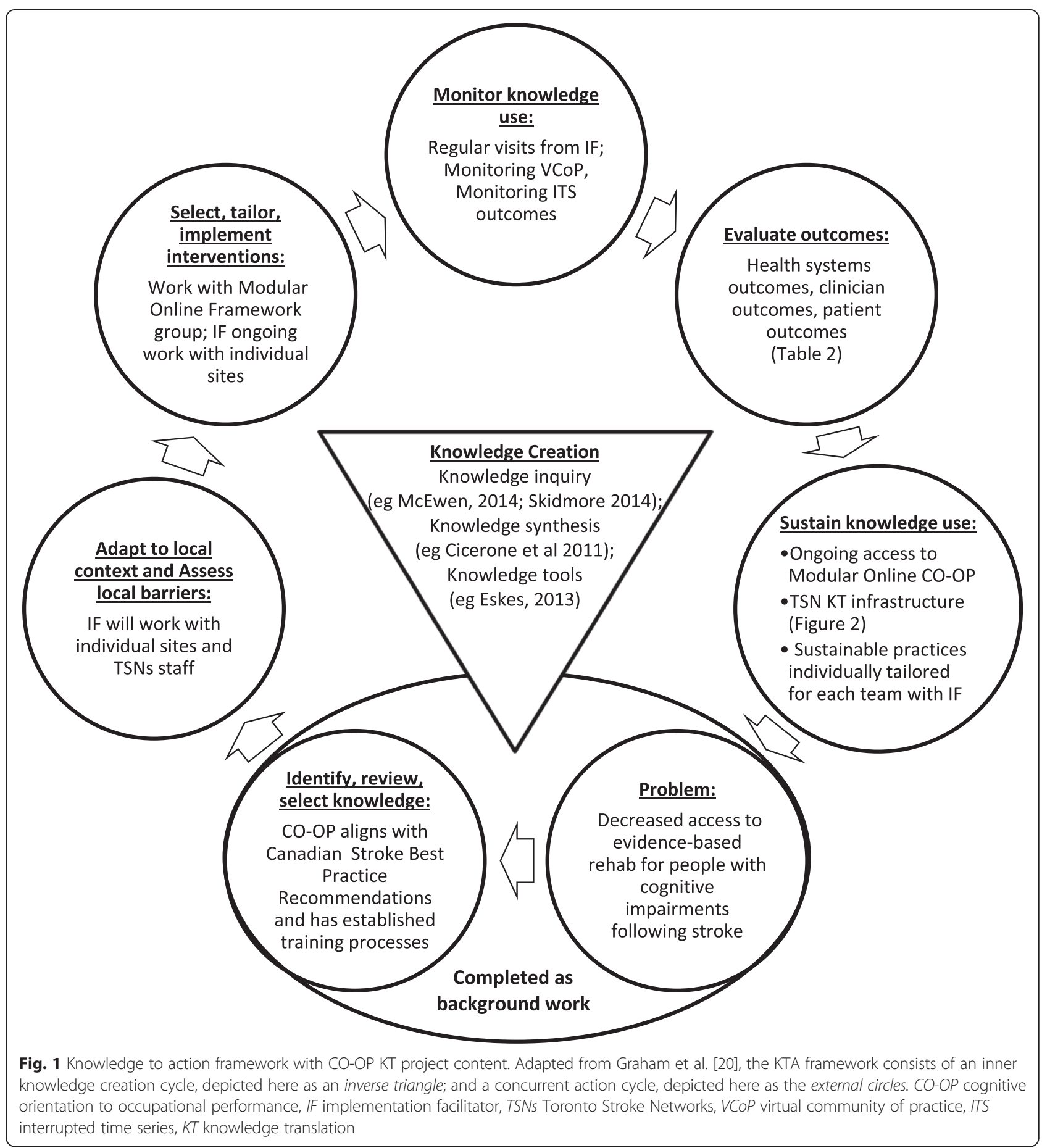

Study 1: admission to rehabilitation

An interrupted time series (ITS) design provides an estimate of the effect of an intervention using a long series of measurements of dependent variables, divided into pre-intervention and post-intervention segments [24]. It is useful when randomized designs are impossible or impractical, as is the case here.
Several years of retrospective rehabilitation referral and admission data are available through the TSNs. Monthly pre-intervention time points will collected for 28 months prior to the intervention implementation period, and post-intervention time points will be collected for 15 months thereafter. Thus, there will be 28 pre-intervention time points, called -T28 (intervention 


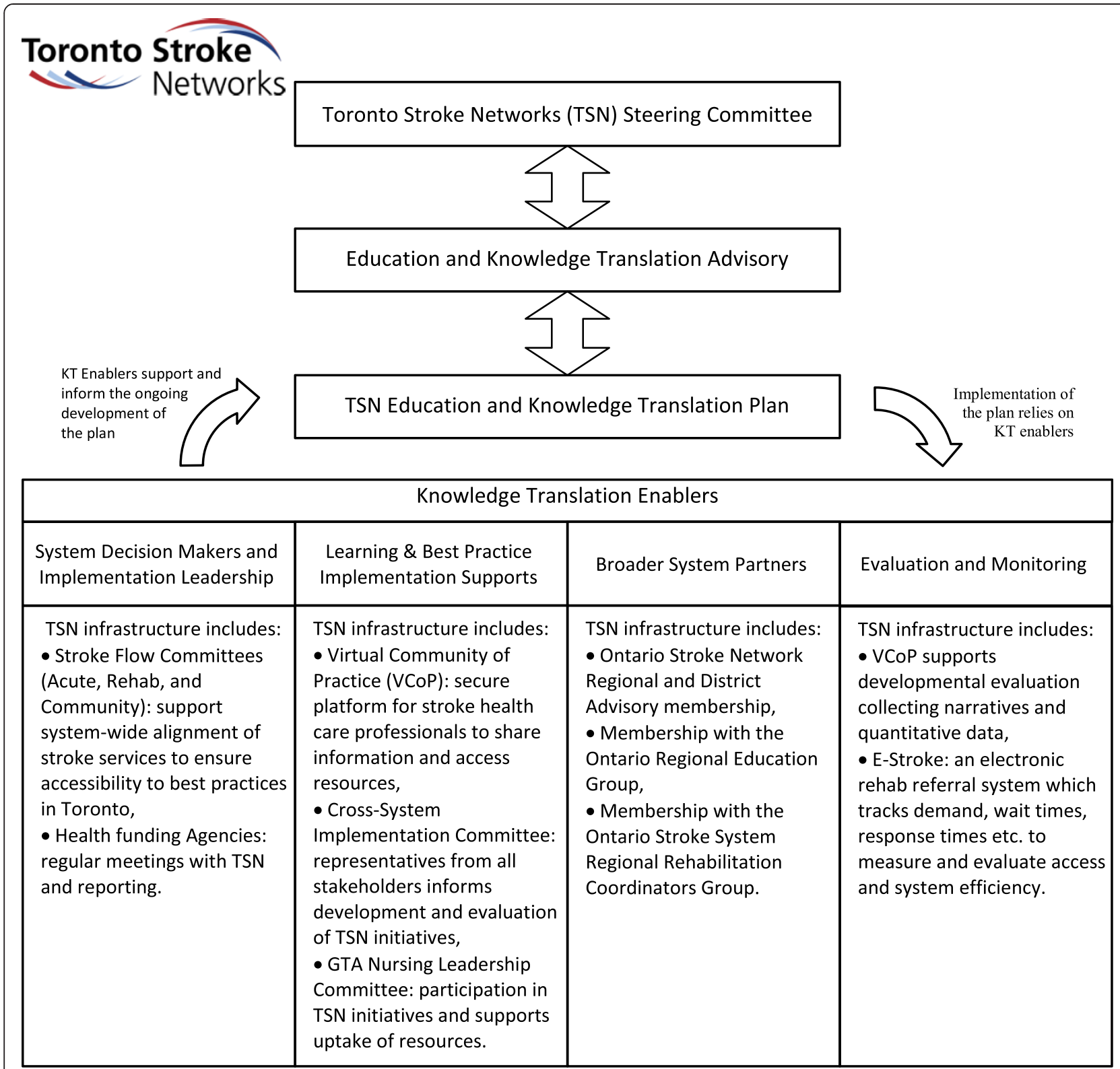

Fig. 2 Toronto stroke networks knowledge translation (KT) infrastructure

implementation minus 28 months), -T27, etc., to -T1. Following - T1, the CO-OP KT intervention will be implemented over a 3-month period. This 3-month intervention implementation period is called T0, during which, no data collection will occur. Next, 15 postintervention monthly time points called $\mathrm{T} 1, \mathrm{~T} 2$, etc., to T15 will occur. Aggregate electronic data will be collected at all pre- and post-intervention time points from the National Rehabilitation Reporting System (NRS) and the TSN's E-Stroke rehab referral system. Data elements will include monthly summaries for the total number of rehabilitation referrals, number of rehabilitation admissions, number of referrals declined, reasons for declined referrals, age, sex, functional independence measure $(\mathrm{FIM})^{\mathrm{Ts}}$ [25] total, motor and cognitive scores of those admitted to rehabilitation, and discharge location.

Study 2: changes in team members' practice, knowledge, and self-efficacy

A pre-post study with a single group of stroke rehabilitation clinicians will be conducted. All stroke rehabilitation clinicians from the five participating sites will be invited to participate, and consenting staff will be enrolled. Indicators of practice change, knowledge change, and self-efficacy are described in Table 1. Chart audits will be used to assess practice change [26], knowledge 
Table 1 Summary of key CO-OP stroke publications

\begin{tabular}{lll}
\hline Authors & Year & Population description \\
\hline $\begin{array}{l}\text { McEwen, S., Polatajko, P., } \\
\text { Huijbregts, M., Ryan, J. }\end{array}$ & $\begin{array}{l}\text { Adults living in the community } \\
\text { with chronic stroke; } 3 \text { single case } \\
\text { experiments }\end{array}$
\end{tabular}

Study design, intervention Main findings

and control

Single case experiments.

Intervention: CO-OP therapy was administered at the rate of one or two sessions per week, up to 10 sessions were completed. Single case paradigm, participants were their own control

McEwen, S., Polatajko, P. Huijbregts, M., Ryan, J.

Skidmore, E., Holm, M., Whyte, E., Dew, M.,

Dawson, D., Becker, J.
2010 Adults with chronic stroke living in the community; 3 single case experiments

2011 Single case admitted to an inpatient rehabilitation unit, cognitively impaired; Age 31; male; time since stroke 7 days
Single case experiments. Intervention: Up to 10 CO-OP sessions were completed. Single case paradigm, participants were their own control

Single case study. Intervention 10 45-min CO-OP sessions. In addition, patient received usual inpatient rehabilitation
Skidmore, E., Dawson, D.,

Whyte, E., Butters, M.,

Dew, M., Grattan, E., Becker, J., Holm, M
2014 Cognitively-impaired patients admitted to an inpatient rehabilitation unit; $\mathrm{N}=10$; mean age: 68; male: $70 \%$; mean time since stroke: 14.5 days; mean length of stay: 22 days
McEwen, S., Polatajko, H., Baum, C., Rios, J., Cirone, D., Doherty, M., Wolf, T.
2014 Patients admitted to an outpatient rehabilitation program; $N=26$; mean age: 56; male: $62.9 \%$; mean time since stroke: 43.3 days; mean number of sessions attended: 12.2 (CO-OP), 13.3 (control). Includes patients with cognitive impairment
RCT. Intervention: CO-OP therapy was administered daily in 30-40 min sessions for the duration of length of stay

Control: dose-matched sessions using scripted and open-ended questions to promote reflection on rehabilitation activities and experiences

Both groups received usual inpatient rehabilitation in addition to the research interventions

RCT. Intervention: CO-OP therapy sessions were $45 \mathrm{~min}$ long and administered twice per week for a maximum of 10 sessions, instead of usual occupational therapy. More complex patients who required additional treatment received additional usual care OT

Control: participants received usual occupational therapy
-PQRS [10] scores showed significant improvement in at least 2/3 self-selected functiona activities, further improvements at 1-month follow-up. Evidence of skill acquisition and retention

-PQRS scores improved for all participants at follow-up in all trained and untrained skills, providing evidence of retention and transfer

-Mean improvement of 6.1 on COPM [16] Performance scale score

-Pittsburgh Rehabilitation participation Scale [measure rehab engagement on 6 point scale] scores improved from 3.2 to 4.9

-FIM ${ }^{\mathrm{TM}}$ [25] scores improved from 68 to 97

-Improvement in self-care skills

-CO-OP participants demonstrated less disability than control participants, FIM $^{\mathrm{TM}}$ 117 vs 96

-CO-OP demonstrated a large effect over usual care on performance of functional activities (PQRS) on trained and untrained activities at follow-up providing evidence of retention and transfer

-CO-OP also demonstrated a medium effect on participation and self-efficacy, compared to usual care

Project team members' names were italicized

$C O-O P$ cognitive orientation to daily occupational performance, $P Q R S$ performance quality rating scale, COPM Canadian occupational performance measure, FIM ${ }^{\mathrm{TM}}$ functional independence measure

tests prepared by $\mathrm{CO}-\mathrm{OP}$ trainers will be used to assess knowledge, and a self-report survey will evaluate changes in self-efficacy. These will be administered immediately before the CO-OP KT intervention (-T1), immediately following (T1), and at a 1-year follow-up (T12). To develop an in-depth understanding of team processes, practices, attitudes related to adoption, and sustainability of best practices for cognitive rehabilitation, site-specific focus groups will be held with consenting clinicians before the intervention (-T1) and at the 1-year follow-up (T12). 
Table 2 Outcomes, indicators, and timing for all studies

\begin{tabular}{|c|c|c|}
\hline Outcomes & Indicators and description & Timing \\
\hline \multicolumn{3}{|c|}{ Study 1: health system: data obtained from electronic referral system, health record, and NRS } \\
\hline Access to inpatient rehabilitation & $\begin{array}{l}\text { Monthly totals: \# of inpatient rehab referrals, \# of admissions, } \\
\text { \# declined; reasons for declined referrals }\end{array}$ & $\begin{array}{l}\cdot-\mathrm{T} 28 \text { to }-\mathrm{T} 1 \\
\cdot \mathrm{T} 1 \text { to } \mathrm{T} 15\end{array}$ \\
\hline \multirow[t]{2}{*}{ Inpatient rehab outcomes } & $\begin{array}{l}\left.\text { Average monthly functional independence measure (FIM }{ }^{\top M}\right) \\
\text { motor and cognitive scores (admission, discharge, and change) }\end{array}$ & $\begin{array}{l}\cdot-\mathrm{T} 28 \text { to }-\mathrm{T} 1 \\
\cdot \mathrm{T} 1 \text { to } \mathrm{T} 15\end{array}$ \\
\hline & $\begin{array}{l}\text { Monthly frequency of discharge locations (home, home with } \\
\text { services, assisted living facility, or acute care) }\end{array}$ & \\
\hline \multicolumn{3}{|c|}{ Study 2: health knowledge: data obtained from stroke rehabilitation team members and chart audits } \\
\hline Rehabilitation team member practice change & $\begin{array}{l}\text { Chart audits will be conducted } 6 \text { months (+/-1 month) before } \\
\text { CO-OP KT implementation as a baseline and to confirm practice } \\
\text { gaps previously identified with interviews [4], and repeated at } \\
6 \text { and } 12 \text { months ( }+/-1 \text { month) following the CO-OP KT intervention. } \\
\text { The chart audit review criteria will center around documentation } \\
\text { of functional goals (e.g., independence with upper body dressing), } \\
\text { rather than impairment-reduction goals (e.g., increase arm strength); } \\
\text { evidence of patient involvement in the goal-setting process; } \\
\text { evidence of teaching of cognitive and problem-solving strategies } \\
\text { as an intervention technique; evidence of use of guided discovery } \\
\text { as an intervention technique }\end{array}$ & $\begin{array}{l}\cdot-\mathrm{T} 6 \\
\cdot \mathrm{T} 6, \mathrm{~T} 12\end{array}$ \\
\hline $\begin{array}{l}\text { Stroke rehab professional self-efficacy with } \\
\text { knowledge and skills related to CO-OP }\end{array}$ & $\begin{array}{l}\text { CO-OP essential elements self-efficacy tool: participants are asked } \\
\text { to rate their ability to perform } 25 \text { elements on a 10-point scale, } \\
\text { with } 1 \text { indicating that they cannot perform the element at all } \\
\text { and } 10 \text { indicating that their performance is excellent. Face validity } \\
\text { evaluated by five members of the International CO-OP Academy }\end{array}$ & $\cdot-\mathrm{T} 1 \cdot \mathrm{T} 1 \cdot \mathrm{T} 12$ \\
\hline $\begin{array}{l}\text { Team perceptions and experiences with } \\
\text { team processes, practices, attitudes related } \\
\text { to adoption and sustainability of best } \\
\text { practices for cognitive rehabilitation }\end{array}$ & $\begin{array}{l}\text { Semi-structured site-specific focus group with groups of 5-8 team } \\
\text { members at a time. Focus groups will be conducted by experienced } \\
\text { facilitator Dr. Anne Hunt who will begin with an open-ended } \\
\text { question "What has been your experience with facilitating recovery } \\
\text { in patients with cognitive impairment?" Based on responses, } \\
\text { Dr. Hunt will probe to obtain a thorough understanding of } \\
\text { perceptions and experiences from a wide range of team } \\
\text { members at each site }\end{array}$ & $\begin{array}{l}\cdot-\mathrm{T} 1 \\
\cdot \mathrm{T} 12\end{array}$ \\
\hline
\end{tabular}

Study 3: health outcomes: data obtained from consenting individual patients

Performance on personally-meaningful, The Canadian occupational performance measure (COPM) is a self-selected activities

Self-efficacy to perform daily activities

Health status

Cognitive screening tool standardized instrument for eliciting performance issues from the client perspective, and for capturing perceived changes in performance over time [16]. The COPM has demonstrated test-retest reliability of 0.89 in people with stroke [34]. A change of 2 points or more on the COPM is considered clinically significant [16]

The self-efficacy gauge (SEG) was designed to measure an individual's self-efficacy in his or her ability to perform daily occupations that span a range of self-care, productivity, and leisure activities. Participants are asked to rate their confidence in their ability to perform 28 items, each on a 10-point scale, with 1 representing "not confident at all" and 10 representing "completely confident". The SEG has very high internal consistency (0.94) and test-retest reliability (0.90) [35]

The stroke impact scale (SIS) [36] is a 59-item questionnaire about the perceived impact of stroke on function and everyday life. The SIS evaluates eight domains. Each item is scored on a 5-point Likert scale related to the degree of difficulty the person with stroke is experiencing. The SIS is widely used in stroke intervention studies as an outcome measure and the psychometric properties of the instrument are well defined [36-38]

The MoCA is a 30-item test of cognitive impairment that includes elements of short-term memory recall; visuospatial capacity; aspects of executive functioning; attention, concentration, and working memory; language; and orientation [29]. The MoCA has excellent internal consistency (0.83) and test-retest reliability (0.92) [29]
- Admission to inpatient rehabilitation

- Discharge from inpatient rehabilitation

- 1 month post discharge

- 3 months post discharge

- 6 months post discharge 


\section{Study 3: patient activity, participation, and self-efficacy outcomes}

To answer question 3, a non-randomized study using historical controls will be conducted. We will compare a group of patients treated by stroke teams who have been exposed to the CO-OP KT intervention (intervention) to a group who have not been exposed (historical control). Prior to the implementation of CO-OP KT, control participants will be recruited, and after the CO-OP KT intervention, intervention participants will be recruited. During the 3-month period when the CO-OP KT intervention is being administered to teams and learning is being consolidated, no recruitment will occur. In addition to data elements universally collected for the ITS Study 1, Study 3 participants will undergo assessments to measure activity performance, participation, and self-efficacy.

Data collection points will occur at the individual patient participants' admission to rehabilitation, discharge from rehabilitation, and at 1,3, and 6 months following discharge. Follow-up assessments will be administered by telephone, a feasible and cost-effective alternative to face-to-face assessments [27]. Data will be collected by family member proxy if the patient participant is not capable of a telephone interview [28]. Table 1 provides a description of all outcomes, indicators, and timing of administration.

\section{Recruitment and sample sizes}

Five inpatient stroke rehabilitation units or combined stroke/neurology units in the TSNs have agreed to participate. Based on past experience, we estimate that the five units together will have approximately 80 admissions per month combined to contribute to the aggregate monthly data. For Study 1 (admission to rehabilitation), we will collect data from all patients aged 18 years or more who have completed inpatient rehabilitation with a primary diagnosis of stroke, defined as Rehabilitation Care Group (RCG) 01.1, 01.2, 01.3, 01.4 or 01.9. For Study 3, a subset of those patients will be recruited and the additional criteria of having at least some cognitive impairment will be applied, determined using the Montreal Cognitive Assessment $($ ) (MoCA@) [29]. The MoCA® is a 30-item test of cognitive impairment that includes elements of short-term memory recall; visuospatial capacity; aspects of executive functioning; attention, concentration, and working memory; language; and orientation. Patients with scores lower than 26 will be included. Additionally, patients will be required to have sufficient English language skills to complete the study assessments (see Table 1 for descriptions). Exclusion criteria are neurological diagnoses other than stroke, presence of major psychiatric illness or capacity issues requiring the use of a substitute decision maker under the Ontario substitute decision maker act. Based on data from a published acute rehabilitation CO-OP study [14], a sample size of 13 per group will have $80 \%$ power to detect a between-group difference of 9 points on the FIM $^{\mathrm{sm}}$, standard deviation of 8 . Allowing for $30 \%$ attrition from all sources, we will recruit 17 participants per group, 34 in total. Based on past projects, we expect a consent rate of one participant per site per month. Thus, recruitment is highly feasible, and will likely be completed in 4 months for each group.

For Study 2 (changes in team members practice, knowledge, and self-efficacy), all clinicians will be invited to participate, and those who provide informed consent will be included in the survey and focus groups. The five stroke teams have approximately 50 staff each, or approximately 250 total. Based on past experience, we expect a participation rate of $50 \%$ for the self-efficacy survey and about $30 \%$ for the focus groups. Therefore, we anticipate running 2 focus groups (1 pre-intervention and 1 post-intervention) with $8-10$ participants each at each of the 5 sites, for a total of 10 focus groups. Chart audits will be conducted on 80 charts of patients discharged approximately 6 months $( \pm 1$ month) before CO-OP KT implementation, and at 6 and 12 months ( \pm 1 month) after CO-OP KT implementation, 240 charts total, providing $89 \%$ power to determine a $25 \%$ change in the audit criteria outlined in Table 1 [30].

\section{Data analysis}

Descriptive statistics will be compiled for all quantitative data collected. The interrupted time series (Study 1) will be analyzed using simulation modeling analysis [31], a trend analysis that provides good power to detect postintervention trend changes when analyzing 30 time points or less per segment. As a precursor to modeling, the time series data will be plotted, analyzed visually for trends, and the degree of autocorrelation will be calculated. For Study 2, pre/post-changes on the clinician self-efficacy survey will be calculated using the paired $t$ test, assuming the data are normally distributed or the signed rank test if not. Qualitative focus group data will be analyzed using directed content analysis [32], with initial codes based on questions derived from the focus group questions.

For Study 3, the non-randomized controlled trial of individual patients, between- and within-group differences on the outcome measures will be examined using repeated measures ANOVA.

\section{Description of the CO-OP KT intervention}

$\mathrm{CO}-\mathrm{OP} \mathrm{KT}$ consists of CO-OP training for the interprofessional team and subsequent implementation support (KT). Two levels of CO-OP training will be provided: (1) A 1 to 2 hour introduction to CO-OP, using an e-learning module and (2) advanced training in the form of a 3-day handson CO-OP workshop. Team members (e.g., occupational 
therapists, physiotherapists, speech-language pathologists, etc.) who are directly involved with teaching the skills for which CO-OP is effective, such as mobility, activities of daily living, or communication, will receive both training components. Team members who are not directly involved in teaching functional skills (e.g., physicians, most nurses, etc.) will be introduced to $\mathrm{CO}-\mathrm{OP}$ using the first introductory elearning module so that they can support and encourage the use of the approach. Funds have been budgeted to cover clinician time when they are participating in either level of training.

KT support based on the Graham et al.'s knowledge to action framework will be provided to facilitate implementation and sustainability of the CO-OP approach [20]. The KT support uses local-adapted knowledge and evidencebased behavior change strategies and will be delivered by an implementation facilitator, to be employed by the project. We will recruit an implementation facilitator who is a health-care professional with experience in both stroke rehabilitation and in implementing educational and knowledge translation initiatives. He or she will make linkages between CO-OP content and existing TSNs KT infrastructure. See Fig. 3 for a detailed visual depiction of existing infrastructure. The implementation facilitator will work closely with project knowledge users MD (regional education coordinator), SQ (regional rehab \& community reengagement coordinator), and SR (clinical nurse specialist). Content will be tailored for the unique environment of each individual team, and the implementation facilitator will be available throughout the project for consultation by telephone or email, will moderate a CO-OP discussion forum on the TSN's online virtual community of practice ( $\mathrm{VCoP})$ website (www.strokecommunity.ca), and will be onsite at each participating institution regularly throughout the project for one-on-one face-to-face coaching and issue resolution. These latter measures will be enacted to meet the knowledge to action framework's requirements of monitoring and sustaining knowledge use.

\section{Sustainability}

Both the sustainability of training and the sustainability of knowledge uptake have been considered. The project has formed a partnership with a group at an educational institution who will develop, implement, and monitor a $\mathrm{CO}-\mathrm{OP}$ e-learning module, ensuring it will be available and updated on a long-term basis. The e-learning module will include both the 1-2 $\mathrm{h}$ introduction to CO-OP and the multi-hour CO-OP workshop. It will provide clinicians who join the inter-professional teams after the CO-OP KT implementation with a means of receiving advanced CO-OP training in a timely and cost-effective manner. Sustainability of uptake will be ensured by Toronto Stroke Networks' KT infrastructure currently in place, as well as through sustainability processes developed locally at each site, in collaboration with the implementation facilitator.

\section{Potential challenges and their solutions}

An issue inherent to time series studies is the possibility that changes not related to the intervention will occur within the system and impact the outcome of interest. By collecting pre-intervention data for 28 months, these trends will be apparent and can be taken into account during analysis. Additionally, time series data will be examined in two ways: we will examine all five units as a group, and also as individual cases. By examining the trends of individual units, any outcome-impacting changes within a single unit, such as a sudden staffing shortage, can be detected and explained.

Influencing practice changes among an entire health system is expected to be challenging. In addition to addressing knowledge, skills, and attitudes of individual clinicians, institutional culture and support for practice change will be significant factors. The implementation facilitator will work together with teams to develop local, site-specific content to help mitigate some of these concerns. Two pragmatic barriers to evidence uptake by health-care professionals are lack of time and lack of resources. We have budgeted for clinician time to attend workshops. Additionally, we have included the position of the implementation facilitator to provide the teams with a human resource with the time and ability to develop materials on their behalf and to provide support while new knowledge and skills are being adopted and

\begin{tabular}{|c|c|c|c|c|c|c|c|c|c|c|c|c|}
\hline Months & $\begin{array}{l}\text { Pre } \\
\text { Study }\end{array}$ & $1-3$ & $4-6$ & $7-10$ & $11-13$ & $14-15$ & $16-18$ & $19-21$ & $22-24$ & $25-30$ & $31-33$ & $34-36$ \\
\hline $\begin{array}{l}\text { Assessment } \\
\text { point }(T)\end{array}$ & $\begin{array}{l}-28 \text { to } \\
-10\end{array}$ & $\begin{array}{c}-9 \text { to } \\
-7\end{array}$ & $\begin{array}{c}-6 \text { to } \\
-4\end{array}$ & -3 to -1 & TO & 1 to 3 & 4 to 6 & 7 to 9 & 10 to 12 & $\begin{array}{c}13 \text { to } \\
15\end{array}$ & \multirow{4}{*}{\multicolumn{2}{|c|}{$\begin{array}{l}\text { Analysis, } \\
\text { reporting }\end{array}$}} \\
\hline Study 1 & \multicolumn{4}{|c|}{ Pre-intervention data collection } & \multirow{3}{*}{ CO-OP KT } & \multicolumn{5}{|c|}{ Post-intervention data collection } & & \\
\hline Study 2 & & & & Baseline & & Post & & & $\begin{array}{l}\text { Follow- } \\
\text { up }\end{array}$ & & & \\
\hline Study 3 & & \multicolumn{3}{|c|}{$\begin{array}{l}\text { Recruit patients: } \\
\text { Historical control }\end{array}$} & & \multicolumn{3}{|c|}{$\begin{array}{l}\text { Recruit patients: } \\
\text { Intervention }\end{array}$} & \multicolumn{2}{|c|}{$\begin{array}{l}\text { Finish follow-up } \\
\text { assessments }\end{array}$} & & \\
\hline
\end{tabular}

Fig. 3 Project design and timeline 
consolidated. The use of the VCoP across sites also provides just-in-time access to peers and experts to support ongoing learning and implementation needs. Access to rehabilitation for persons with cognitive impairment has been identified as a priority by the TSNs acute and rehab stroke flow task groups, with representation from decision-makers across Toronto organizations. These decision-makers have made a collective commitment to local implementation and to supporting this project across the system.

Patient recruitment among the stroke patient population is challenging [33]. To mitigate this and reduce the burden on point of care clinicians, our inclusion criteria are broad and we have dedicated research assistant time for recruitment and retention.

The implementation facilitator will monitor CO-OP intervention fidelity to ensure that the rehabilitation team members are adopting $\mathrm{CO}-\mathrm{OP}$ appropriately. $\mathrm{He}$ or she will observe at least one video recorded treatment session from each team member who has taken the advanced $\mathrm{CO}-\mathrm{OP}$ workshops and rate their use of $\mathrm{CO}-\mathrm{OP}$ using an existing $\mathrm{CO}-\mathrm{OP}$ treatment fidelity checklist (http://ot.utoronto.ca/coop). This will also act as a feedback and instructional mechanism. The implementation facilitator will keep a journal of all implementation activities, successes, and challenges as the project unfolds. As well, regular reporting on the local status of CO-OP implementation will occur to Toronto Stroke Networks decision-makers through existing channels.

\section{Trial status}

Not yet recruiting.

\section{Ethics}

The study received lead site Research Ethics Board approval from the Sunnybrook Health Sciences Centre Human Research Protections Program (PIN 177-2015).

\section{Discussion}

This project will advance general knowledge about the degree to which the implementation of a supported, integrated, inter-professional KT initiative can sustainably change health system outcomes (access to rehabilitation), knowledge outcomes (rehabilitation team practice), and patient outcomes (functional improvement). Additionally, it will advance knowledge about the degree to which changes can be sustained over the longer term through integrated KT mechanisms and committed partnerships with health and academic institutions.

\section{Competing interests}

The authors declare that they have no competing interests.

\section{Authors' contributions}

SM and EL co-led the team building and protocol development. SM was the primary author of the protocol. EL has contributed substantially to the protocol, particularly to aspects related to the Toronto Stroke Networks. She has overseen the project development as a whole. MD has contributed to overall writing, the development of the CO-OP KT intervention, and the description of the existing $\mathrm{KT}$ infrastructure. DD consulted on cognitive impairment and cognitive rehabilitation, ME on knowledge translation, and $\mathrm{AH}$ on goal-setting and health professional behavior. SQ has consulted on community engagement, and SR has consulted on aspects of the project related to incorporating nursing. All authors have reviewed and approved the manuscript.

\section{Author details}

${ }^{1}$ Sunnybrook Research Institute, Toronto, Canada. ${ }^{2}$ University of Toronto, Toronto, Canada. ${ }^{3}$ Sunnybrook Health Sciences Centre, Toronto, Canada. ${ }^{4}$ Rotman Research Institute, Baycrest, Toronto, Canada. ${ }^{5}$ University of Ottawa, Ottawa, Canada. ${ }^{6}$ Bloorview Research Institute and Holland Bloorview Kids Rehab Hospital, Toronto, Canada.

Received: 15 October 2015 Accepted: 29 October 2015

Published online: 05 November 2015

\section{References}

1. Sharp S, Linkewich E, Willems J, Tahair N, Levy C, Bayley M. What should really be happening to our stroke patients post-acute care? A system best practice model for inpatient rehab [abstract]. Stroke. 2014;45:ATP259.

2. Cicerone KD, Langenbahn DM, Braden C, Malec JF, Kalmar K, Fraas $M$, et al. Evidence-based cognitive rehabilitation: updated review of the literature from 2003 through 2008. Arch Phys Med Rehabil. 2011;92(4):519-30.

3. Melkas $\mathrm{S}$, Jokinen $\mathrm{H}$, Hietanen M, Erkinjuntti T. Poststroke cognitive impairment and dementia: prevalence, diagnosis, and treatment. Degener Neurol Neuromuscul Dis. 2014;4:21-7.

4. Linkewich E. Cognitive rehab: a grounding in what is and what could be. 2014.

5. Polatajko HJ, Mandich A. Enabling occupation in children: the cognitive orientation to daily occupational performance (CO-OP) approach: first edition ed. Ottawa: CAOT Publications ACE; 2004

6. Eskes $\mathrm{G}$, on behalf of the Canadian Stroke Best Practices and Standards Working Group. Mood and cognition in patients following stroke. In: Lindsay MP, Gubitz G, Bayley M, Phillips S, editors. Canadian stroke best practice recommendations. 4th ed. Ottawa: Canadian Stroke Network; 2013.

7. Toronto Stroke Networks. http://www.tostroke.com (2013). Accessed October 13, 2015.

8. Ankolekar S, Renton C, Sare G, Ellender S, Sprigg N, Wardlaw JM, et al. Relationship between poststroke cognition, baseline factors, and functional outcome: data from "efficacy of nitric oxide in stroke" trial. J Stroke Cerebrovasc Dis. 2014;23(7):1821-9.

9. Health Quality Ontario \& Ministry of Health and Long-Term Care. Qualitybased procedures clinical handbook for stroke. 2013.

10. McEwen SE, Polatajko HJ, Huijbregts MP, Ryan JD. Exploring a cognitive-based treatment approach to improve motor-based skill performance in chronic stroke: results of three single case experiments. Brain Inj. 2009;23(13-14):1041-53.

11. McEwen SE, Polatajko HJ, Huijbregts MPJ, Ryan JD. Inter-task transfer of meaningful, functional skills following a cognitive-based treatment: results of three multiple baseline design experiments in adults with chronic stroke. Neuropsychol Rehabil. 2010;20(4):541-61.

12. McEwen S, Polatajko H, Baum C, Rios J, Cirone D, Doherty M, et al. Combined cognitive-strategy and task-specific training improve transfer to untrained activities in subacute stroke: an exploratory randomized controlled trial. Neurorehabil Neural Repair. 2015;29(6):526-36.

13. Skidmore ER, Holm MB, Whyte EM, Dew MA, Dawson D, Becker JT. The feasibility of meta-cognitive strategy training in acute inpatient stroke rehabilitation: case report. Neuropsychol Rehabil. 2011;21(2):208-23.

14. Skidmore ER, Dawson DR, Whyte EM, Butters MA, Dew MA, Grattan ES, et al. Developing complex interventions: lessons learned from a pilot study examining strategy training in acute stroke rehabilitation. Clin Rehabil. 2014:28(4):378-87.

15. Polatajko HJ, McEwen SE, Ryan JD, Baum CM. Pilot randomized controlled trial investigating cognitive strategy use to improve goal performance after stroke. Am J Occup Ther. 2012;66(1):104-9. 
16. Law M, Babtiste S, Carswell A, McColl MA, Polatajko H, Pollock N. Canadian occupational performance measure. 5th ed. Ottawa: CAOT Publications ACE; 2014.

17. Davis $D$, Evans $M$, Jadad A, Perrier L, Rath $D$, Ryan $D$, et al. The case for knowledge translation: shortening the journey from evidence to effect. BMJ. 2003;327(7405):33-5.

18. National Collaborating Centre for Methods and Tools. Knowledge translation initiatives using a systems approach. Hamilton: McMaster University; 2013

19. Kitson AL. The need for systems change: reflections on knowledge translation and organizational change. J Adv Nurs. 2009;65(1):217-28.

20. Graham ID, Logan J, Harrison MB, Straus SE, Tetroe J, Caswell W, et al. Lost in knowledge translation: time for a map? J Contin Educ Health Prof. 2006;26(1):13-24

21. Canadian Interprofessional Health Collaborative. A national interprofessional competency framework. 2010.

22. Dawson DR, Gaya A, Hunt A, Levine B, Lemsky C, Polatajko HJ. Using the cognitive orientation to occupational performance (CO-OP) with adults with executive dysfunction following traumatic brain injury. Can J Occup Ther. 2009;76(2):115-27

23. Henshaw E, Polatajko H, McEwen S, Ryan JD, Baum CM. Cognitive approach to improving participation after stroke: two case studies. Am J Occup Ther. 2011;65(1):55-63.

24. Penfold RB, Zhang F. Use of interrupted time series analysis in evaluating health care quality improvements. Acad Pediatr. 2013;13(6 Suppl):S38-44.

25. Keith RA, Granger CV, Hamilton BB, Sherwin FS. The functional independence measure: a new tool for rehabilitation. Adv Clin Rehabil. 1987:1:6-18.

26. Foy R, Eccles MP. Audit and feedback interventions. In: Straus SE, Tetroe J, Graham ID, editors. Knowledge translation in health care: moving from evidence to practice. Chichester: John Wiley \& Sons, Ltd; 2013.

27. Korner-Bitensky N, Wood-Dauphinee S, Siemiatycki J, Shapiro S, Becker R Health-related information postdischarge: telephone versus face-to-face interviewing. Arch Phys Med Rehabil. 1994;75(12):1287-96.

28. Segal ME, Schall RR. Determining functional/health status and its relation to disability in stroke survivors. Stroke. 1994;25(12):2391-7.

29. Nasreddine ZS, Phillips NA, Bedirian V, Charbonneau S, Whitehead V, Collin I, et al. The Montreal cognitive assessment, MoCA: a brief screening tool for mild cognitive impairment. J Am Geriatr Soc. 2005;53(4):695-9.

30. StatsToDo. Computer Program to Calculate Sample Requirement to Compare 2 Proportions. https://www.statstodo.com/SSiz2Props_Pgm.php. Accessed October 13, 2015

31. Borckardt JJ, Nash MR, Murphy MD, Moore M, O'Neil P. Clinical practice as natural laboratory for psychotherapy research. Am Psychol. 2008;63(2):77-95.

32. Hsieh HF, Shannon SE. Three approaches to qualitative content analysis. Qual Health Res. 2005;15(9):1277-88.

33. Blanton S, Morris DM, Prettyman MG, McCulloch K, Redmond S, Light KE, et al. Lessons learned in participant recruitment and retention: the EXCITE trial. Phys Ther. 2006;86(11):1520-33.

34. Cup EH, Scholte op Reimer WJ, Thijssen MC, van Kuyk-Minis MA. Reliability and validity of the Canadian occupational performance measure in stroke patients. Clin Rehabil. 2003;17(4):402-9.

35. Gage M, Noh S, Polatajko HJ, Kaspar V. Measuring perceived self-efficacy in occupational therapy. Am J Occup Ther. 1994;48(9):783-90.

36. Duncan PW, Wallace D, Lai SM, Johnson D, Embretson S, Laster LJ. The stroke impact scale version 2.0. Evaluation of reliability, validity, and sensitivity to change. Stroke. 1999;30(10):2131-40.

37. Duncan PW, Bode RK, Min Lai S, Perera S, Glycine Antagonist in Neuroprotection Americans Investigators. Rasch analysis of a new stroke-specific outcome scale: the stroke impact scale. Arch Phys Med Rehabil. 2003;84(7):950-63.

38. Edwards $\mathrm{B}, \mathrm{O}^{\prime}$ Connell $\mathrm{B}$. Internal consistency and validity of the stroke impact scale 2.0 (SIS 2.0) and SIS-16 in an Australian sample. Qual Life Res. 2003;12(8):1127-35.

\section{Submit your next manuscript to BioMed Central and take full advantage of:}

- Convenient online submission

- Thorough peer review

- No space constraints or color figure charges

- Immediate publication on acceptance

- Inclusion in PubMed, CAS, Scopus and Google Scholar

- Research which is freely available for redistribution

Submit your manuscript at www.biomedcentral.com/submit 